\title{
Optimal design of functionally graded material columns for buckling problems
}

\author{
N. T. Alshabatat \\ Department of Mechanical Engineering, King Abdulaziz University-Rabigh, SA \\ Department of Mechanical Engineering, Tafila Technical University, Tafila, Jordan \\ Phone: +966583079470 \\ Email: nabee1963030@yahoo.com
}

\begin{abstract}
This paper presents a method for improving the buckling capacity of slender columns by employing functionally graded materials (FGMs) instead of isotropic materials in constructing these members. The volume fractions of FGM constituents are varied along the column length by a trigonometric function thereby causing variations in material properties such as stiffness and density. The effective material properties are evaluated based on Mori-Tanaka scheme. The buckling problem has been solved using the finite element method (FEM) whereas optimal solution was obtained through a genetic algorithm. The present design problem considered identification of the optimal volume fraction distribution of the FGM that maximizes the critical buckling load-to-weight ratio for columnar members with different boundary conditions. The different design examples presented in this paper illustrate the effectiveness of using FGMs in constructing axially compressed columns. The present results can be successfully applied in designing FGM-columns for optimal buckling capacities.
\end{abstract}

Keywords: Axially graded columns; column buckling; structural optimization; genetic algorithms; finite element.

\section{INTRODUCTION}

Axially compressed elastic columns are widely used in mechanical and civil engineering. If sufficiently slender, these members would normally fail due to buckling. The buckling critical load depends on the boundary conditions, elastic modulus, cross-sectional second moment of area, and length. Usually, increasing the critical load can be achieved by selecting a material with higher modulus of elasticity and/or increasing the column's second moment of area. These, in turn, are expected to result in an increase in the weight of the column. A critical challenge becomes obtaining excellent buckling properties while controlling the weight of the specific member under consideration, i.e., achieving a high buckling-capacity-to-weight ratio. Several studies were carried out on improving the buckling capacity of columns using various techniques including shape modifications [1,2] and material tailoring [3,4]. In this study, functionally graded material is used to maximize the buckling capacity of elastic columns subjected to different boundary conditions.

FGMs are composite materials with spatially continuous variation of material properties. These properties include moduli of elasticity, Poisson's ratio, density, etc. The continuous variation of properties can be realized by the continuous variation in material composition. This is normally achieved by continuously varying the volume fractions of its compositional constituents throughout the volume or along a specific direction of interest. In contrast to laminate composite materials where both the structure and properties change in a stepwise manner the properties of FGMs change 
smoothly through the volume or the specific direction of interest in which case the problems of flaws delamination associated with the laminated structures are eliminated. The FGMs can be used, in a controlled manner, to improve the performance of structures by controlling compositional variations thereby controlling particular properties of interest. The idea of FGMs was initially presented in Japan in the 1980s through the spaceplane mission in which the materials used were needed to endure large temperature deviations. Different techniques are developed for producing FGM such as powder technology and centrifugal casting. More details about the engineering of FGMs may be found somewhere else [5,6].

Buckling properties of FGMs columns were investigated in several studies. For example, Singh and Li [7] proposed a low dimensional model for evaluating the buckling critical loads of FG columns in the longitudinal direction. FGM columns were approximated by another type of columns with piecewise unvarying material properties using closed-form transcendental functions. The buckling capacities of rectangular and annular FGM columns were investigated in comparison with homogenous columns by Heydari [8]. The conservation of energy principle was used to obtain the stability equations. The variation calculus method was used to get the critical buckling load and dimensionless first mode shape for simply supported and clamped boundary conditions. The relationships between the critical buckling loads of a FGM Timoshenko beam and that of equivalent homogeneous Euler-Bernoulli beam exposed to compression was studied analytically by $\mathrm{Li}$ and Batra [9]. They showed that the critical buckling load can be evaluated based on equivalent homogeneous Euler-Bernoulli beam in addition to some coefficients related to variation in mechanical properties through-the-thickness. Using the localized differential quadrature method, Yilmaz et al. [10] studied the buckling of axially FG columns with elastic restraints using the localized differential quadrature method. The buckling behaviors of axially FG and non-uniform Timoshenko beam were investigated by Huang et al. [11]. The coupled governing equations were converted into a system of linear algebraic equations having a non-trivial solution utilizing auxiliary function and power series. The characteristic polynomial equations were directly derived and the lower and higher-order eigenvalues were calculated simultaneously from the multi-roots. Based on the first-order shear deformation theory, Kahya and Turan [12] investigated the buckling of FGM columns using a new finite element consisting of five nodes with two degrees of freedom at each node. The material properties were varied through the beam thickness according to a simple powerlaw. The finite element stiffness matrices were estimated using Lagrange's method. Aldadah et al. [3] studied the buckling of inhomogeneous columns by dividing the columns into small segments with uniform section and modulus of elasticity for each one. The microstructure of the column consisted of two materials with different elastic moduli. A Monte Carlo technique was employed to create the microstructures of each segment at arbitrary volume fractions. After creating the microstructure, the eigenvalue problem was solved numerically using linear perturbation analysis which is provided by the commercial software ABAQUS. They obtained an analytical form for the ensemble averaged critical buckling load based on exhausted simulation results at arbitrary volume fractions. Possible designs for the highest and lowest buckling loads have been obtained. Buckling of slender columns using FG microstructures was investigated by Ranganathan et al. [4]. The modulus of elasticity along the column length was varied in a controlled way to find the variation that optimizes the critical buckling load in comparison with a reference homogeneous column. The eigenvalue problems were solved using linear perturbation analysis and Rayleigh-Ritz method.

The main objective of the present work is to design lightweight FGM columns with enhanced buckling capacity. In particular, the design objective is to find the maximum specific buckling load (i.e., load/weight ratio) of columns using the concept of FGMs with axial gradients. The columns under considerations have the same dimensions and different boundary conditions. The finite element method is used to model the FGM columns and to solve the eigenvalue/eigenvector problem. The optimal material distributions are determined using a genetic algorithm optimizer. 


\section{THEORETICAL BACKGROUND}

\section{Functionally Graded Material}

Consider a column made of functionally graded material which is a mixture of two constituents. The volume fractions of constituent materials are assumed to vary continuously along the longitudinal coordinate (i.e., $x$ coordinate). Thus, the material properties of the FGM column such as the modulus of elasticity and the density vary in the longitudinal coordinate according to the volume fractions of the two constituents. The volume fractions of constituent $1\left(V_{1}\right)$ and constituent $2\left(V_{2}\right)$ have ranges between zero and one (i.e., $0 \leq V_{1} \leq 1$ and $\left.V_{2}=1-V_{1}\right)$.

This paper suggested that the volume fractions through the length of FGM column follow a fourparameter trigonometric law [13]:

$$
V_{l}(x)=\left[\frac{1}{2}-\frac{\alpha}{2} \sin \left(\frac{\eta \pi x}{L}+\phi\right)\right]^{\gamma}
$$

where $\alpha, \eta, \phi$, and $\gamma$ are the parameters which control the volume fraction variation throughout the longitudinal direction of column. These parameters must be selected such that $V_{l}$ will be bounded between zero and one. Equation 1 provides a flexible description of material volume fraction profile which gives a great tool for design optimization [13].

The local effective material properties of FGM columns are estimated based on volume fraction distribution of the constituents. The homogenization method of Mori and Tanaka [14,15] is applied to estimate the effective mechanical properties at a point $x$ along the column length. Other micromechanical models for obtaining FGM effective properties can be found in Alshabatat and Naghshineh [16]. In Mori-Tanaka method, the modulus of elasticity at a point can be calculated using

$$
E(x)=\frac{9 K(x) G(x)}{3 K(x)+G(x)}
$$

Where $K(x)$ and $G(x)$ are the effective local bulk and the effective local shear moduli at any point $x$, respectively. These moduli can be calculated using

$$
\frac{K(x)-K_{1}}{K_{2}-K_{1}}=\frac{V_{2}(x)}{1+\frac{3 V_{1}(x) *\left(K_{2}-K_{1}\right)}{3 K_{1}+4 G_{1}}}
$$

and

$$
\frac{G(x)-G_{1}}{G_{2}-G_{1}}=\frac{V_{2}(x)}{1+\frac{V_{1}(x) *\left(G_{2}-G_{1}\right)}{G_{1}+f_{1}}}
$$

where

$$
f_{1}=\frac{G_{1}\left(9 K_{1}+8 G_{1}\right)}{6\left(K_{1}+2 G_{1}\right)}
$$

The subscripts 1 and 2 in the previous equations refer to constituent 1 and constituent 2 , respectively. The mass density $\rho$ at any point in the FGM plate is obtained by the rule of mixtures

$$
\rho(x)=\rho_{1} V_{1}(x)+\rho_{2} V_{2}(x)
$$


where $\rho_{1}$ and $\rho_{2}$ are the densities of constituent 1 and constituent 2 , respectively. The finite element method is used to model the FGM column. The length of the column is divided into equal lengths elements. Each finite element has two nodes and six degrees of freedom. The finite element was modeled as a homogeneous material with properties assigned according to the effective properties at its centroid.

\section{Buckling Analysis}

The buckling loads for axially loaded column can be found from the following differential equation:

$$
E(x) I \frac{\partial^{2} w}{\partial x^{2}}+P_{c r} w=0
$$

where $I$ is the moment of inertia of column cross section, $w$ is the transverse deflection, and $P_{c r}$ is the critical buckling load. Due to the complexity of the current problem, an analytical solution might not be achievable. Therefore, the finite element method is employed at this point in order to achieve the buckling analysis of the FGM columns. The finite element method gives the following system of linear equations [17]

$$
\operatorname{det}\left([K]-\lambda\left[K_{\sigma}\right]\right)=0
$$

where $[K]$ is the global stiffness matrix, $\left[K_{\sigma}\right]$ is the global stress stiffness matrix, and the smallest root $\lambda$ represents the smallest buckling load of the column (i.e., these equations are solved as an eigenvalue/eigenvector problem). The accuracy of the finite element program was validated through a comparison of the current results with other studies [4] and [7]. The columns under consideration are $\mathrm{CF}, \mathrm{CC}$, and SS columns, where the symbol (C) represents a clamped end, and the symbol (F) represents a free end, and the symbol (S) represents a simply-supported end as shown in Fig. 1. An example of a finite element mesh of a column is shown in Fig. 2. The column is divided into 10 equal finite elements. Each element has two nodes and each node has axial, transverse, and rotational degrees of freedom.

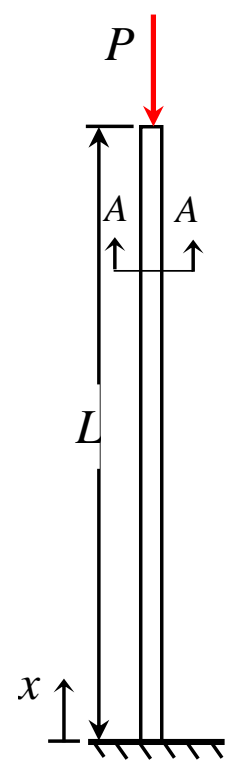

(a)

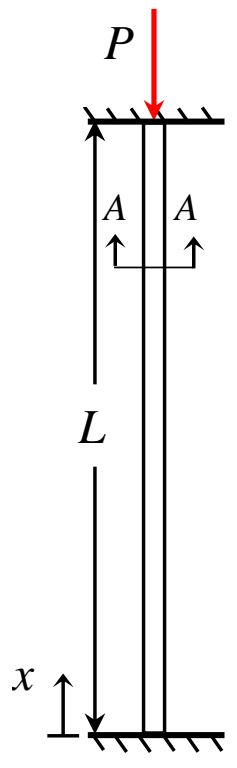

(b)

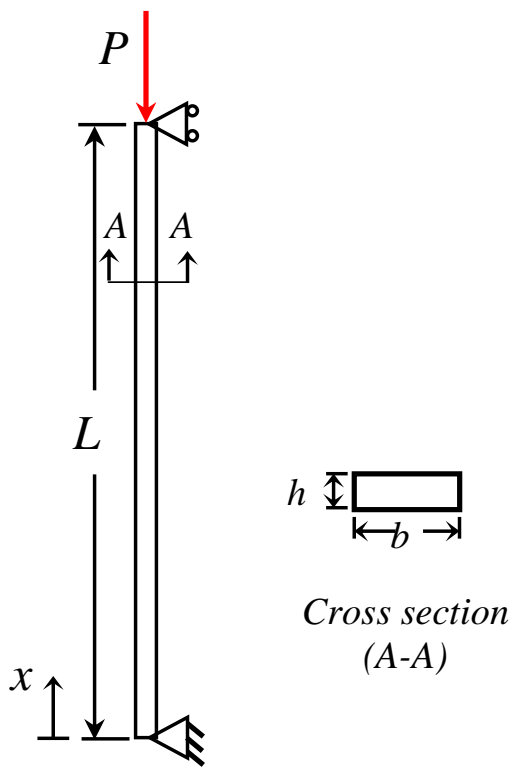

(c)

Figure 1. Schematic diagrams of (a) CF column, (b) CC column, (c) SS column, and (d) cross section $(A-A)$. 
The benchmark problems studied the column axial gradient using linear function (i.e., $E(x)=E_{o}(1+x / L)$ ) and a quadratic function (i.e., $\left.E(x)=E_{o}(1+x / L)^{2}\right)$. The buckling load was normalized as $\bar{P}=P_{c r} L^{2} / E_{o} I$. The comparison results, which are summarized in Tables 1 and 2 , show a very good agreement between the current results and those reported in the literature with extremely small differences; percent differences between the current results, with 15 elements, and the results of [7] is less than $0.1 \%$ and $0.3 \%$ for linear and quadratic gradient, respectively.

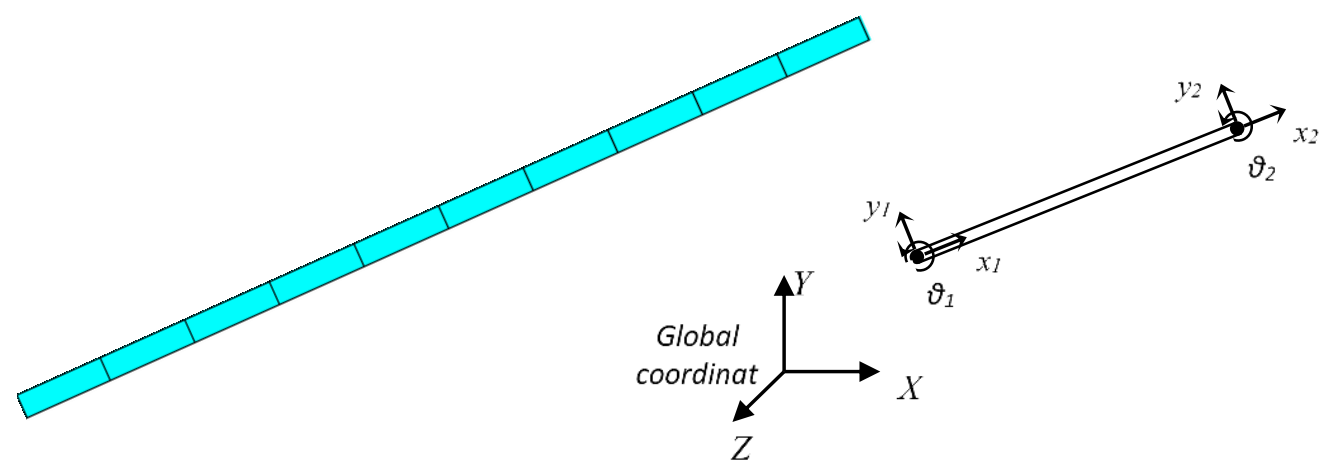

(a)

(b)

Figure 2. (a) Finite element model for a column, and (b) 6 DOF beam element.

Table 1. Normalized buckling load $\bar{P}$ for linear axial gradient.

\begin{tabular}{|c|c|c|c|c|c|}
\hline \multirow{3}{*}{ Column Type } & \multicolumn{5}{|c|}{ Normalized critical buckling load $\bar{P}$} \\
\hline & \multicolumn{3}{|c|}{ Present (FEM) } & \multirow{2}{*}{ Ref. [4] } & \multirow{2}{*}{ Ref. [7] } \\
\hline & 5 elements & 10 elements & 15 elements & & \\
\hline Clamped-Free & 3.138 & 3.123 & 3.120 & --- & 3.123 \\
\hline Clamped-Clamped & 57.826 & 57.459 & 57.419 & --- & 57.445 \\
\hline Simply-Supported & 14.489 & 14.505 & 14.509 & 14.515 & 14.505 \\
\hline
\end{tabular}


Table 2. Normalized critical buckling load $\bar{P}$ for quadratic axial gradient.

\begin{tabular}{|c|c|c|c|c|c|}
\hline \multirow{3}{*}{ Column Type } & \multicolumn{5}{|c|}{ Normalized critical buckling load $\bar{P}$} \\
\hline & \multicolumn{3}{|c|}{ Present (FEM) } & \multirow{2}{*}{ Ref. [4] } & \multirow{2}{*}{ Ref. [7] } \\
\hline & 5 elements & 10 elements & 15 elements & & \\
\hline Clamped-Free & 3.895 & 3.851 & 3.843 & --- & 3.858 \\
\hline Clamped-Clamped & 83.201 & 82.143 & 82.012 & --- & 82.228 \\
\hline Simply-Supported & 20.679 & 20.765 & 20.780 & 20.836 & 20.792 \\
\hline
\end{tabular}

\section{Optimization Using Genetic Algorithm}

In this paper, genetic algorithm (GA) is used in designing the FGM columns. The GA is an optimization technique employing the concept of biological progression [18]. The GA is started with a set of candidate points (called population) forming the first generation or iteration (i.e., the set of four design variables in Eq. (1) form a single candidate point in a search space). This population covers the whole search space.

The objective function (fitness function) is then evaluated for each candidate point in this population. The points with worthy fitness functions are used to create a new population using three operators: reproduction, crossover, and mutation. The new population forms the second generation (or iteration). The algorithm continues over many generations till a termination criterion has been met, such as reaching a specified maximum number of generations, or the change in the best objective function between generations is smaller than a specified percentage. The basic steps of the GA can be described in the flowchart shown in Fig. 3. 


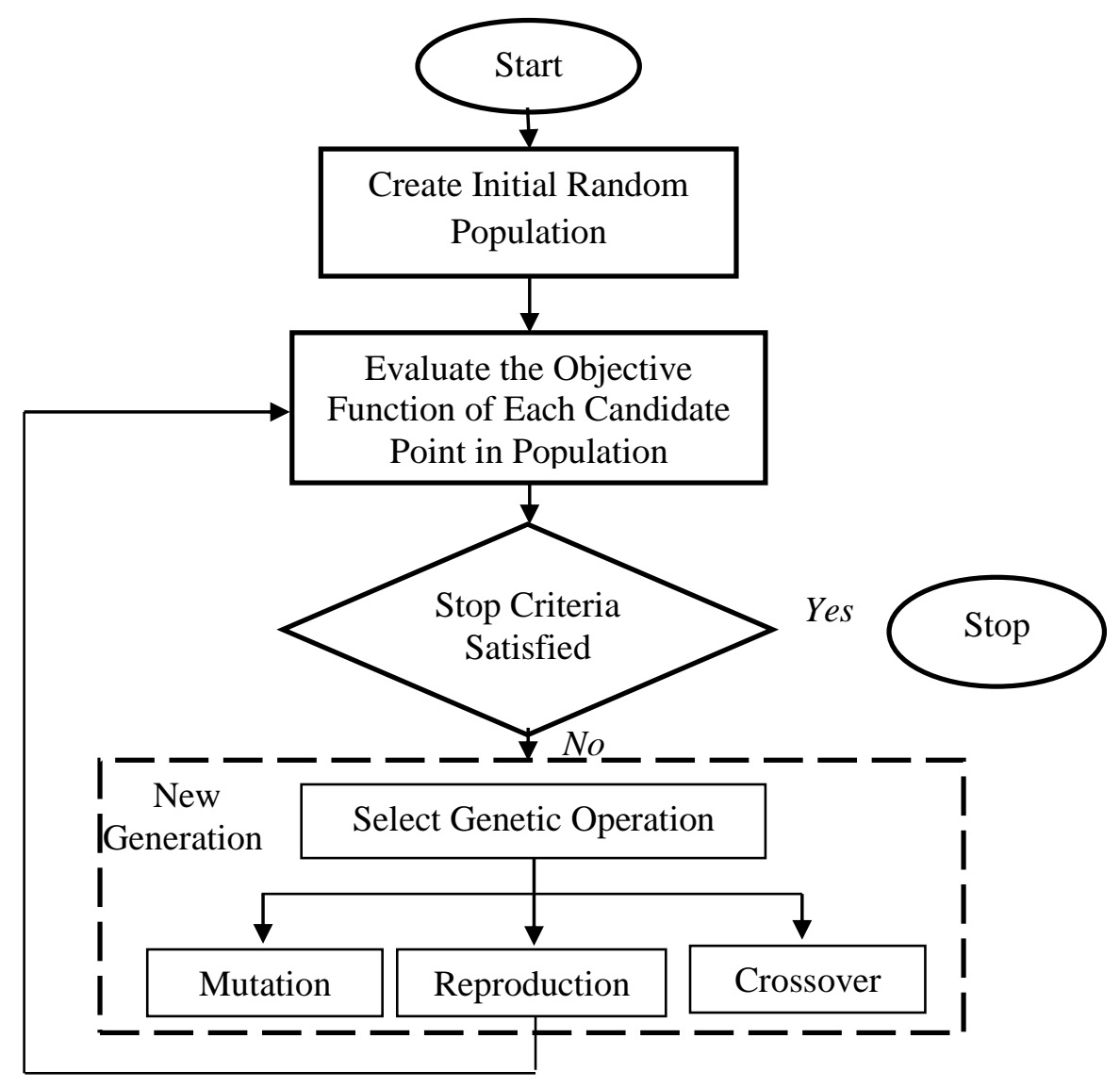

Figure 3. Flowchart for GA optimization procedure.

\section{COLUMN DESIGN EXAMPLES}

The present design problem seeks maximizing the lowest critical buckling load-to-weight ratio of columns using FGM. The considered FGM is composed of zirconia $\left(\mathrm{ZrO}_{2}\right)$ and aluminum $(\mathrm{Al})$. The properties of $\mathrm{ZrO}_{2}$ and aluminum are summarized in Table 3. The optimal design of the FGM column is achieved by optimizing the volume fractions of the material constituents along its length. In other words, we look for the best values of parameters $\alpha, \eta, \phi$, and $\gamma$ in the trigonometric law distribution (see Eq. 1) which give the optimal design of the column. The optimization problem can be summarized as

$$
\begin{aligned}
& \text { Maximize }\left(\frac{P_{c r, I}}{W}\right) \\
& \text { Subject to: } 0 \leq V_{1} \leq 1 \\
& \alpha_{\text {min }} \leq \alpha \leq \alpha_{\max } \\
& \eta_{\min } \leq \eta \leq \eta_{\max } \\
& \phi_{\min } \leq f \leq \phi_{\max } \\
& \gamma_{\min } \leq \gamma \leq \gamma_{\max }
\end{aligned}
$$

Throughout this study, $V_{1}$ is the zirconia constitute and the bounds of design variables are selected as: $\alpha_{\text {min }}=0, \alpha_{\max }=1, \eta_{\min , \max }= \pm 10, \phi_{\min , \max }= \pm \pi, \gamma_{\min }=0$, and $\gamma_{\max }=20$. The columns under considerations have the same dimensions (i.e., $L=1 \mathrm{~m}, b=0.05 \mathrm{~m}$, and $h=0.01 \mathrm{~m}$ ) and different boundary conditions. Genetic Algorithm is used as an optimization tool. The population size for 
each generation is selected to be 100. Successive generations are determined by reproducing two elite individuals from the previous generation. Of the remaining 98 individuals to be determined, 75 are produced by using a scattered crossover, and remaining 23 of individuals are determined by mutation, where a random number taken from a Gaussian distribution is added to each entry of the parent individual.

Table 3. Material properties of zirconia $(\mathrm{ZrO} 2)$ and aluminum $(\mathrm{Al})$.

\begin{tabular}{ccc}
\hline Property & Zirconia $\left(\mathrm{ZrO}_{2}\right)$ & Aluminum \\
\hline$E(\mathrm{GPa})$ & 205 & 70 \\
$\rho\left(\mathrm{kg} / \mathrm{m}^{3}\right)$ & 6050 & 2700 \\
\hline
\end{tabular}

The optimum design parameters which maximize the first buckling load-to-weight ratio are shown in Table 4 for CF, CC, and SS columns. The results illustrated in Table 4, show that the buckling load-to-weight ratio of the optimum FGM columns are more than those of isotropic $\mathrm{ZrO}_{2}$ and $\mathrm{Al}$ columns. With respect to the buckling load-to-weight ratio of aluminum columns, the increase is more than $40 \%$ for all the FGM columns with different boundary conditions.

Shown in Figs. 4-6 are the optimal FGM profiles that yield the greatest critical load-to-weight ratio for various boundary conditions. These results can be explained by comparing the volume fraction distributions with bending moment diagrams. The curvature of FGM column under compression load can be given by [8]

$$
\kappa(x)=\frac{M(x)}{E_{F G}^{*} I}
$$

where $\kappa(x)$ and $M(x)$ are the curvature and bending moment as functions of $x$-coordinate, and $E_{F G}^{*}$ is a parameter depends on the volume fraction distribution and the moduli of elasticity of the FGM constituents. For small lateral deflection, Eq. 10 can be written as:

$$
E_{F G}^{*} I \frac{d^{2} y}{d x^{2}}=M(x)
$$

Table 4: The optimal design parameters of columns which maximize the critical buckling load-toweight ratio

\begin{tabular}{lccccccc}
\hline \multirow{2}{*}{ Column Type } & \multicolumn{5}{c}{$P_{c r} / W$} & \multicolumn{5}{c}{ FGM parameters } \\
\cline { 2 - 8 } & $\mathrm{ZrO} 2$ & $A l$ & $F G M$ & $\alpha$ & $\eta$ & $\phi$ & $\gamma$ \\
\hline Clamped-Free & 71.0 & 54.4 & 78.7 & 1 & 1.087 & -1.889 & 0.698 \\
Clamped- Clamped & 1136.3 & 869.4 & 1254.4 & 1 & 4.175 & -1.847 & 0.613 \\
& & & & & & & \\
Simply-Supported & 284.1 & 217.4 & 313.8 & 1 & 2.115 & 1.406 & 0.487 \\
\hline
\end{tabular}


In the first example, buckling of CF column was considered. The deflection of this column is $y(x)=y_{\max }(1-\cos (\pi x / 2 L))$. Substituting $y(x)$ into Eq. 11 and double differentiating yields an expression for the bending moment

$$
M(x)=M_{\max } \cos \left(\frac{\pi x}{2 L}\right)
$$

The bending moment of CF column is shown in Figure 4(b). It is clear from Figure 4 that placing zirconia (which is stiffer than aluminum) near the location of high bending moment and placing aluminum (which is lighter than zirconia) near the location of low bending moment significantly increases the critical load-to-weight ratio.

In the second example, buckling of CC column was considered. The deflection of this column is $y(x)=y_{\max }(1 / 2-(1 / 2) \cos (2 \pi x / L))$. Substituting $y(x)$ into Eq. 11 and double differentiating yields an expression for the bending moment

$$
M(x)=M_{\max } \cos \left(\frac{2 \pi x}{L}\right)
$$

The bending moment of CC column is shown in Figure 5(b). In this case, the maximum values of the bending moment occur near the ends and the middle of the column. This would explain the optimal volume fraction distribution of zirconia.

In the third example, buckling of SS column was considered. The deflection of this column is $y(x)=y_{\max } \sin (\pi x / L)$. Substituting $y(x)$ into Eq. 11 and double differentiating yields an expression for the bending moment

$$
M(x)=M_{\max } \sin \left(\frac{2 \pi x}{L}\right)
$$

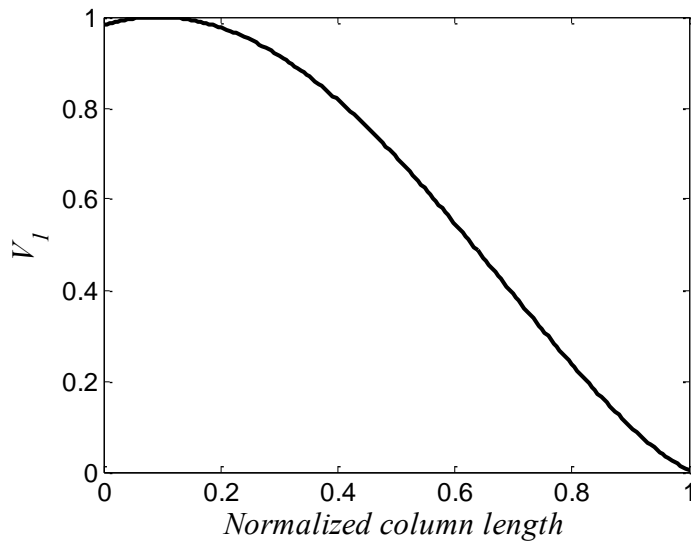

(a)

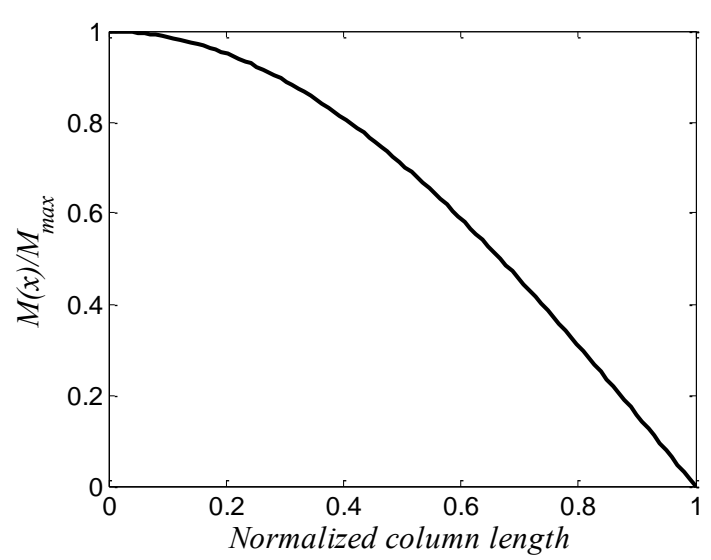

(b)

Figure 4. (a) Zirconia volume fraction optimal distribution of a CF-FGM optimal column, and (b) Bending moment diagram of a CF column. 


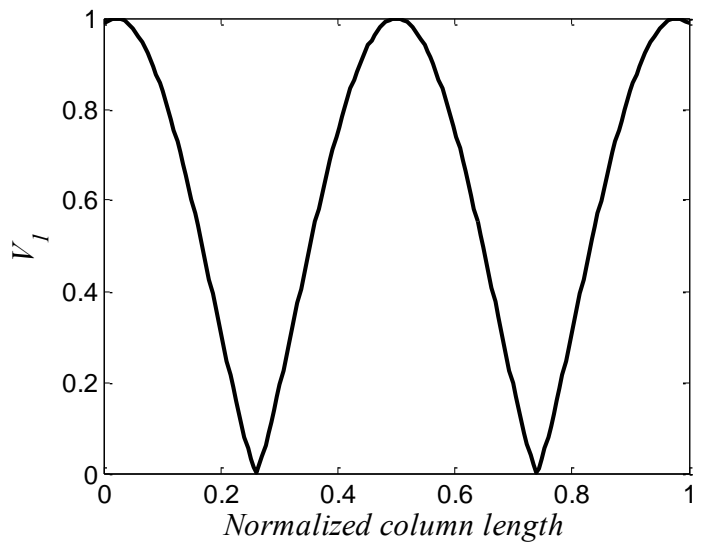

(a)

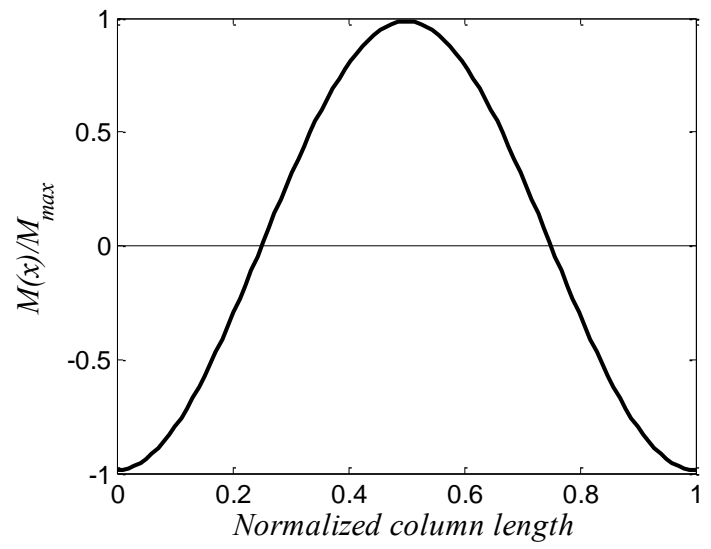

(b)

Figure 5. (a) Zirconia volume fraction optimal distribution of a CC-FGM optimal column, and (b) Bending moment diagram of a $\mathrm{CC}$ column.

The bending moment of SS column is shown in Figure 6(b). In this case, the maximum values of the bending moment occur near the middle of the column. Again, we can conclude the same about the optimal volume fraction distribution of zirconia. On the whole, an important physical nature is revealed in the optimum profiles: placing stiff material (like zirconia) near the locations of high bending moment increases significantly the critical load-to-weight ratio. Also, it is beneficial to have a light material (like aluminum) at other places.

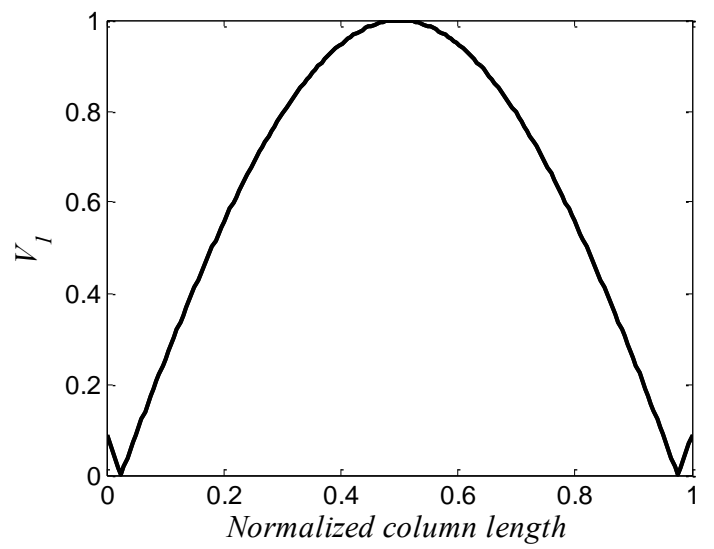

(a)

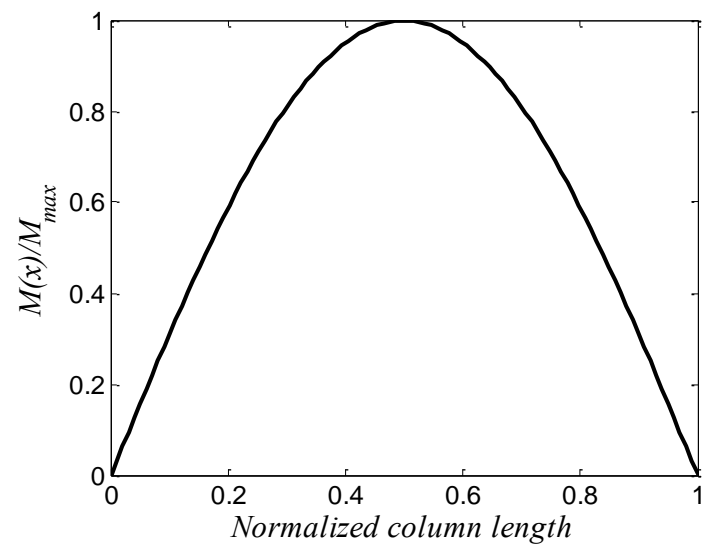

(b)

Figure 6. (a) Zirconia volume fraction optimal distribution of a SS-FGM optimal column, and (b) Bending moment diagram of a SS column.

In addition, the columns buckling mode shapes were studied to see if the optimal columns exhibited mode shapes that were significantly different from those of a homogeneous column. The first buckling mode shapes of homogeneous and optimal FGM columns are shown in Figs. 6-8. 


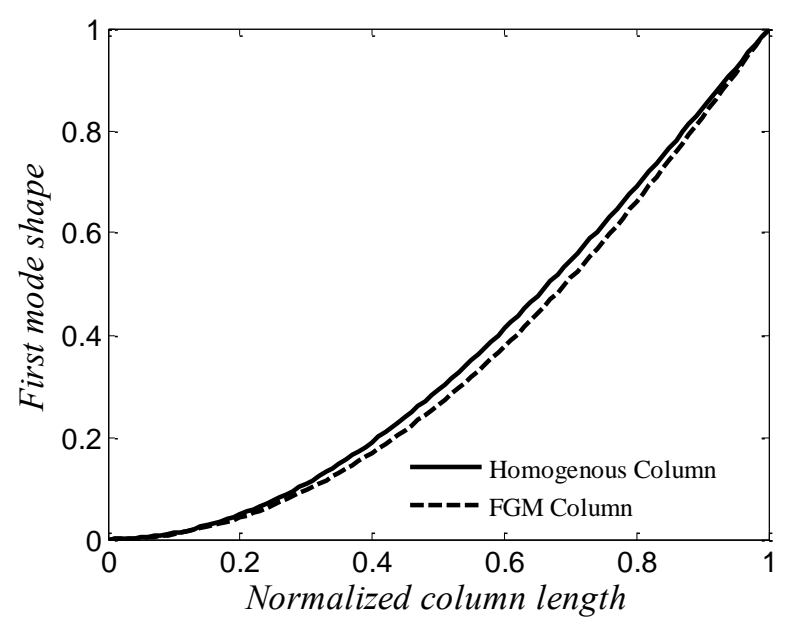

Figure 6. The normalized mode shapes of CF columns.

The modal assurance criterion (MAC) is used to examine the difference in mode shapes between homogeneous and optimal columns. The MAC is commonly used in the area of vibration and structural acoustics $[16,19]$ and is given by

$$
M A C_{H, F G}=\frac{\left(\left\{\phi_{H}\right\}^{T}\left\{\phi_{F G}\right\}\right)^{2}}{\left(\left\{\phi_{H}\right\}^{T}\left\{\phi_{H}\right\}\right)\left(\left\{\phi_{F G}\right\}^{T}\left\{\phi_{F G}\right\}\right)}
$$

where $\left\{\phi_{H}\right\}$ is the buckling mode of the homogeneous column, and $\left\{\phi_{F G}\right\}$ is the buckling mode of the optimal FGM column. A MAC value of 1.0 indicates that the two buckling modes are identical. The MAC values for all the boundary conditions are greater than 0.998 which mean that no major changes occurred in the columns buckling mode shapes.

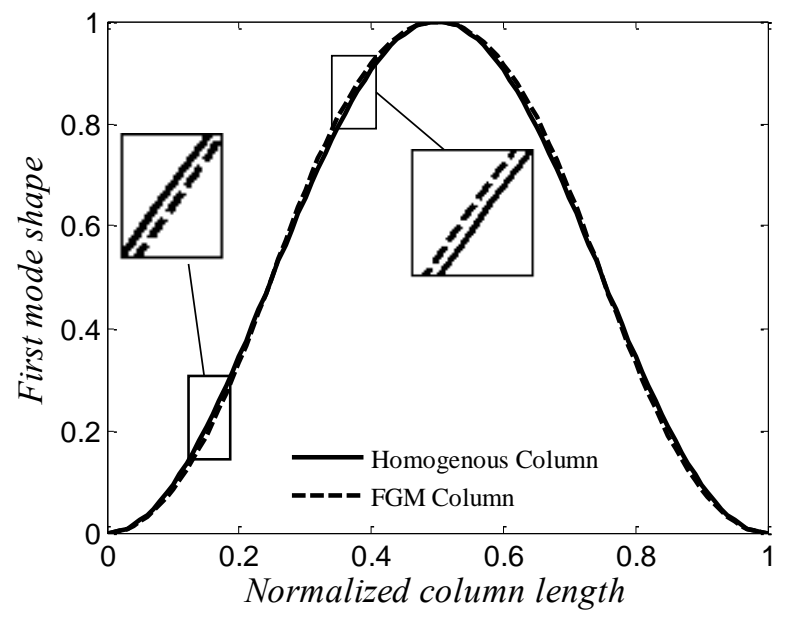

Figure 7. The normalized mode shapes of CC columns. 


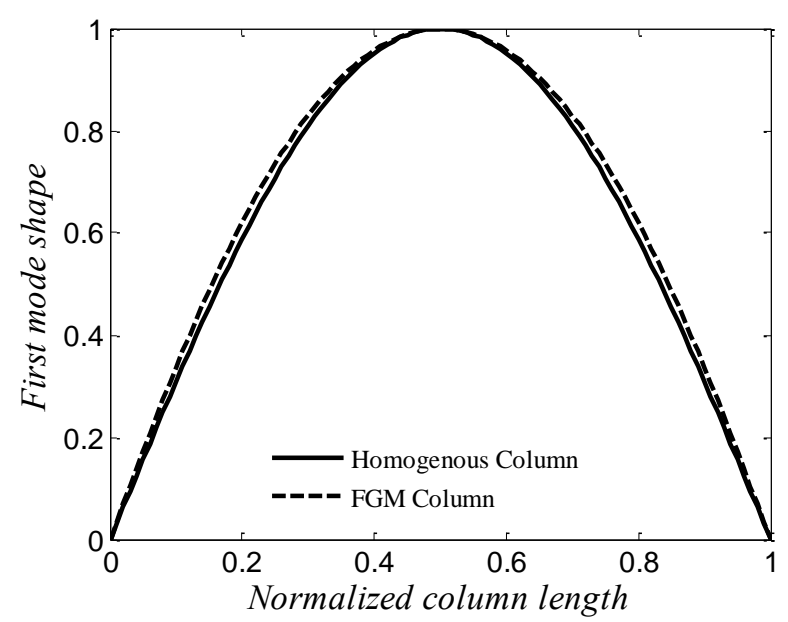

Figure 8. The normalized mode shapes of SS columns.

\section{CONCLUSION}

This article demonstrates the efficiency of using FGMs for designing axially compressed columns. A trigonometric functional distribution of material volume fraction throughout column length is considered to maximize the buckling capacity. Finite element method was used to solve the eigenvalue/eigenvector problem, and GA was used as an optimization tool to find the optimal design parameters in the trigonometric law. The finite element code was tested via benchmark results available in the literature. The design method was applied to find the maximum first buckling load-to-weight ratio for clamped-free, clamped-clamped, and simply-supported FGM columns. The numerical results showed that the compositional gradient through column length can be effectively used to enhance the design of axially compressed elastic columns. It has been shown that concentrating the constituent with higher stiffness near the locations of high bending moment increases significantly the critical buckling load-to-weight ratio and it is helpful to have a lighter constituent at other places. Future extensions of this work would include the optimal design of thick columns with material gradients through both the thickness and the length. In addition, manufacturing constraints should be taken in consideration in fabricating FGM-columns.

\section{REFERENCES}

[1] Manickarajah D, Xie YM, Steven GP. Optimization of columns and frames against buckling. Computers and Structures. 2000;75:45-54.

[2] Maalawi KY, El-Chazly NM. Practical shapes of the strongest columns. Journal of Engineering and Applied Science. 2004;51:543-58.

[3] Aldadah MG, Ranganathan SI, Abed FH. Buckling of two phase inhomogeneous columns at arbitrary phase contrasts and volume fractions. Journal of Mechanics of Materials and Structures. 2014; 9:465-474.

[4] Ranganathan SI, Abed FH, Aldadah MG. Buckling of slender columns with functionally graded microstructures. Mechanics of Advanced Materials and Structures. 2016;23:13601367.

[5] Miyamoto Y, Kaysser W, Rabin B. Functionally Graded Materials: Design, Processing, and Applications. Springer. 1999; New York.

[6] Rasheedat M, Akinlabi ET, Shukla M, Pityana S. Functionally graded material: An overview. World Congress on Engineering. 2012. 
[7] Singh KV, Li G. Buckling of functionally graded and elastically restrained non-uniform columns. Composites: Part B. 2009;40:393-403.

[8] Heydari A. Buckling of functionally graded beams with rectangular and annular sections subjected to axial compression. International Journal of Advanced Design and Manufacturing Technology. 2011;5:25-31.

[9] Li SR, Batra R. Relations between buckling loads of functionally graded Timoshenko and homogeneous Euler-Bernoulli beams. Composite Structures. 2013;95:5-9.

[10] Yilmaz Y, Girgin Z, Evran S. Buckling analyses of axially functionally graded nonuniform columns with elastic restraint using a localized differential quadrature method. Mathematical Problems in Engineering. 2013;2013:1-12.

[11] Huang Y, Zhang M, Rong H. Buckling analysis of axially functionally graded and nonuniform beams based on Timoshenko theory. Acta Mechanica Solida Sinica. 2016;29:200207.

[12] Kahya V, Turan M. Finite element model for vibration and buckling of functionally graded beams based on the first-order shear deformation theory. Composites: Part B. 2017;109:108115.

[13] Alshabatat N, Naghshineh K. Optimization of natural frequencies and sound power of beams using functionally graded material. Advances in Acoustics and Vibration. 2014; 2014:1-10.

[14] Mori T, Tanaka T. Average stress in matrix and average elastic energy of materials with misfitting inclusions. Acta Metallurgica. 1973;21:571-574.

[15] Benvensite Y. A new approach to the application of Mori-Tanaka's theory in composite materials. Mechanics of Materials. 1987;6:147-157.

[16] Alshabatat N, Myers K, Naghshineh K. Design of in-plane functionally graded material plates for optimal vibration performance. Noise Control Engineering Journal. 2016;64:268-278.

[17] Cook R, Malkus D, Plesha M. Concepts and applications of finite element analysis. John Wiley \&Sons. New York. 2002.

[18] Holland J. Adaptation in natural and artificial systems. University of Michigan Press. Michigan. 1975.

[19] Erwins DJ, Modal testing: theory and practice. Brül \& Kjær. Denmark. 1986. 